\title{
Modeling of XUV-induced damage in Ru films: the role of model parameters
}

\author{
Igor Milov, ${ }^{1, *}$ (1) Vladimir Lipp, ${ }^{2}$ (i) Nikita Medvedev, ${ }^{3,4}$ (i) Igor A. MaKhotKin, ${ }^{1}$ \\ Eric LoUis, ${ }^{1}$ and Fred BIJKerk ${ }^{1}$ \\ ${ }^{1}$ Industrial Focus Group XUV Optics, MESA + Institute for Nanotechnology, University of Twente, Drienerlolaan 5, 7522 NB Enschede, \\ The Netherlands \\ ${ }^{2}$ Center for Free-Electron Laser Science CFEL, Deutsches Elektronen-Synchrotron DESY, Notkestrasse 85, Hamburg 22607, Germany \\ ${ }^{3}$ Institute of Physics, Czech Academy of Sciences, Na Slovance 2, 18221 Prague 8, Czech Republic \\ ${ }^{4}$ Institute of Plasma Physics, Czech Academy of Sciences, Za Slovankou 3, 18200 Prague 8, Czech Republic \\ *Corresponding author: i.milov@utwente.nl
}

Received 4 June 2018; revised 27 August 2018; accepted 27 August 2018; posted 28 August 2018 (Doc. ID 334228); published 28 September 2018

\begin{abstract}
We perform a computational study of damage formation in extreme ultraviolet (XUV)-irradiated ruthenium thin films by means of combining the Monte Carlo approach with the two-temperature model. The model predicts that the damage formation is most affected by ultrafast heating of the lattice by hot electrons, and is not very sensitive to the initial stage of the material excitation. Numerical parameters of the model were analyzed, as well as different approximations for the thermal parameters, showing the importance of the temperature dependence of the electron thermal conductivity and the electron-phonon coupling factor. Our analysis reveals that the details of photoabsorption and ultrafast non-equilibrium electron kinetics play only a minor role in the XUV irradiation regime. () 2018 Optical Society of America
\end{abstract}

OCIS codes: (140.3330) Laser damage; (140.2600) Free-electron lasers (FELs); (340.7480) X-rays, soft x-rays, extreme ultraviolet (EUV); (160.3900) Metals; (240.0310) Thin films.

\section{INTRODUCTION}

Survivability of optical elements exposed to ultrafast (femtosecond) high-peak-power free-electron laser (FEL) pulses becomes more and more important in the context of rapidly developing extreme ultraviolet (XUV) and x-ray FEL light sources [1-5]. Such sources are capable of generating pulses with energies sufficiently high to cause significant damage of the optics used at these facilities. A fundamental understanding and accurate description of the processes responsible for the degradation of optics are required in order to increase their lifetime.

The quality of modeling of the interaction of ultrafast high-intensity laser pulses with matter strongly depends on the proper choice of model parameters. Despite the fact that the field of ultrafast laser-matter interaction has been extensively developed in recent decades, reliable thermal parameters such as electron heat capacity, electron thermal conductivity, and electron-phonon coupling factor are still unknown for many materials relevant for XUV and x-ray optics. The knowledge of such parameters, and especially their electron temperature dependence in the regime of strong laser excitation, is crucial in order to get a quantitatively correct description of the processes involved. In the regime of high electron temperature, all the mentioned parameters can significantly differ from their room-temperature values [6]. The use of temperaturedependent thermal parameters for simulations of the electron temperature evolution in a highly excited gold target in Ref. [7] led to a good agreement with experimental findings, while lowtemperature values of the parameters failed to reproduce experimental data. In another work, a modification of the common expression for thermal conductivity in order to take into account d-band excitation enabled the authors to explain the details of melting dynamics in silver [8]. Measuring and calculating material properties in the regime of strong laser excitation when the electron temperature reaches values much higher than the Fermi temperature is challenging and requires separate dedicated studies.

Our work focuses on ruthenium $(\mathrm{Ru})$ as a prospective material for XUV [9] and x-ray [10-12] grazing incidence reflective mirrors. The relatively high value of its critical angle $\left(27^{\circ}\right.$ at $92 \mathrm{eV}$ photon energy) allows operation in a wide range of grazing incidence angles. In our recent study [13], we investigated femtosecond XUV-induced single-shot damage of a $50 \mathrm{~nm}$ thick $\mathrm{Ru}$ film on a $\mathrm{Si}$ substrate. The experiment was performed at Free-electron LASer in Hamburg (FLASH) [1]. Experimental analysis of the damaged morphologies, together with simulations of photoabsorption and ultrafast evolution of 
electron and lattice temperatures, showed that the nature of single-shot damage is photomechanical spallation, a phenomenon that was previously studied in the case of irradiation of solids with optical [14-19] and XUV [20-23] lasers. The spallation threshold for Ru was measured to be $F_{\text {spal }}=200 \pm$ $40 \mathrm{~mJ} / \mathrm{cm}^{2}$ of incident fluence [13].

In this paper, we analyze the model parameters of $\mathrm{Ru}$ as a material that is still poorly studied in the field of ultrafast lasermatter interaction despite its promising applications as just mentioned. The evolution of electron and lattice temperatures is calculated with our combined approach using the XCASCADE(3D) Monte Carlo (MC) code [24,25] and the two-temperature model (TTM) [26,27]. The influence of a particular choice of model parameters on the results of our calculations is investigated in order to determine which parameters play the most important role in the phenomenon of XUV single-shot damage.

\section{MODEL}

The interaction of a femtosecond XUV laser pulse with a metal starts with photoabsorption. Depending on the photon energy and the atomic constituents of the target, a photon may be absorbed by the conduction band or core atomic shells with ionization potentials lower than or equal to the photon energy. The photon attenuation length in $\mathrm{Ru}$ for the case of normal incidence, as a function of the photon energy, is shown in Fig. 1. The figure compares photon attenuation lengths from Refs. [28-30]. The referenced datasets agree for photon energies above $\sim 200 \mathrm{eV}$, but in the range from $\sim 50$ to $200 \mathrm{eV}$ there is some uncertainty in the data. At lower energies, the data from Refs. $[28,29]$, calculated within the atomic approximation, qualitatively diverge from the experimental data on solid $\mathrm{Ru}$ from Ref. [30]. This indicates that at energies $<50 \mathrm{eV}$, collective effects such as the band structure of $\mathrm{Ru}$ and plasmon excitations start to play an important role. In this work, we focus on the photon energy of $92 \mathrm{eV}$, as was used in experiments at FLASH (see Ref. [13]). At this photon energy, there is almost no difference between the experimental data and data from Ref. [29], so either of them can be used, although one

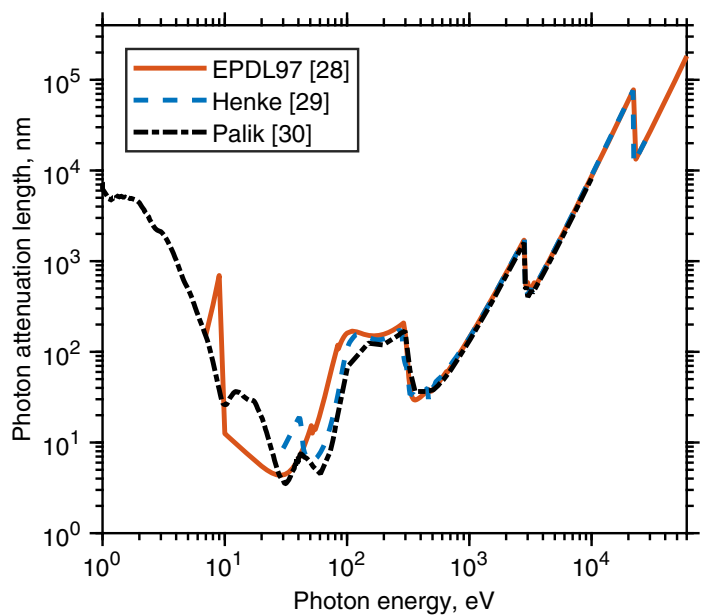

Fig. 1. Dependence of photon attenuation length on photon energy in $\mathrm{Ru}$ at normal incidence conditions. has to keep in mind that the photon attenuation length is only known with some uncertainty for this energy. At photon energies where there is a significant difference, the experimental data from Ref. [30] must be taken.

As was shown in previous studies, an electronic system of a solid under femtosecond FEL irradiation follows the so called "bump-on-hot-tail" distribution [31,32]: the majority of lowenergy electrons is almost in thermal equilibrium, with the minority of the highly excited electrons forming the highenergy out-of-equilibrium tail of the distribution. This typical shape allows one to split the electron distribution into the high-energy and low-energy fractions, and treat each of them individually with appropriate methods. Note that a proper interconnection between the fractions (and, correspondingly, the methods) must be made in order to obtain reliable results.

\section{A. XCASCADE(3D)}

The absorption of XUV photons and the non-equilibrium high-energy electron kinetics induced as a result of photoabsorption is simulated with an event-by-event MC code $\mathrm{XCASCADE}(3 \mathrm{D})$ [24,25]. The code models a target as a homogeneous arrangement of atoms (atomic approximation) with a density corresponding to the chosen material. The photoabsorption cross sections and the ionization potentials of the target are also described in the atomic approximation. The model applicability is limited to a particular range of photon energies. The lower limit of $50 \mathrm{eV}$ is due to solid-state effects, whereas the upper limit is defined by relativistic effects, which give an error of $\sim 10 \%$ at the energy of $40 \mathrm{keV}$, or $\sim 20 \%$ at the energy of $100 \mathrm{keV}$.

The code accounts for the following processes: photoabsorption by deep-shell levels, Auger recombination of created holes with release of an Auger electron, propagation of photo- and secondary electrons, and inelastic (impact ionization) and elastic scattering of electrons on neutral atoms [24,25]. Within the MC event-by-event simulation model, both ballistic and diffusive regimes of electron transport appear naturally, and the transition between the two is automatically accounted for based on the kinetic energy of the electrons [25]. All photo- as well as secondary electrons are traced until their energy falls below a predefined cutoff energy. Electrons with energies below this cutoff, as well as holes created in the valence atomic levels, are considered as thermalized and belonging to the bath of the conductionband electrons. Their energy is treated as the energy of the conduction-band electrons, as will be discussed in more detail.

XCASCADE(3D) takes advantage of the approximation of non-interacting electrons. The free electron-electron scattering is neglected, so the cascades develop independently. Such an approximation is valid if the density of high-energy electrons participating in the cascading process is significantly smaller than the atomic density, thereby making impact ionization and elastic scattering the dominant processes of electron interaction $[33,34]$. In other words, it means that the fluence of an incident laser pulse must not be too high to produce a density of excited electrons comparable to or higher than atomic density of a target.

Within the XCASCADE(3D) code we also assume that the material properties are not affected during the electron 
cascading, i.e., the photoionization, impact ionization, and elastic scattering cross sections do not change significantly due to excitation of the target. Consequently, the cross sections for the unexcited material are applied. This approximation implies that all processes that significantly change the material electronic or atomic structure, such as melting or vaporization, must occur after the electron cascading is finished. In the case of femtosecond FEL pulses, this assumption is consistent with the low-fluence approximation made previously.

The inelastic scattering resulting in impact ionization is modeled with the binary-encounter-Bethe cross section [35], whereas for the elastic scattering, the Mott's cross section with Moliere screening parameter is employed [34], both valid within the atomic approximation. The cross sections neglect collective effects within the target, which play a role at electron energies below $\sim 50 \mathrm{eV}$. Although we use the same cross sections for electrons with lower energies, this should not influence the kinetics much, since electrons with such energies reach the cutoff after only a few collisions [36].

When all photo- and secondary electrons lose their energy to a level below the cutoff energy, the cascading stops. At this point we consider the electronic system to be thermalized to the Fermi-Dirac distribution. A possible deviation of the lowenergy electrons from the Fermi-Dirac distribution is not taken into account. This would require dedicated simulations with, e.g., the Boltzmann equation $[37,38]$. It is expected that within the bump-on-hot-tail distribution, such deviations are small.

To calculate the number and energy density depth profiles, we perform the simulation in two steps: first, we perform simulations for bulk material assuming all photons are absorbed at $z=0$ ("surface"). Then, the realistic absorption positions are taken into account by applying a convolution with the Lambert-Beer's law. Here we treat the region $z \geq 0$ as irradiated material, while region $z<0$ is considered as vacuum above the surface. This approach enables one to estimate the total energy emitted from the sample, but not the actual distribution of energy above the surface. The units of energy density, eV/atom, are used for the vacuum region to be compared with the energy density inside the material, although there are no atoms in vacuum; this should only be used to estimate the total emitted energy, and can be converted into the energy density units of, e.g., $\mathrm{eV} / \mathrm{cm}^{3}$ by multiplying with the target atomic density under normal conditions.

\section{B. Two-Temperature Model}

The low-energy electrons are used as the energy source in the TTM. The transport of energy within this formalism is diffusive in nature since the standard Fourier law of heat conduction is used. Such an assumption breaks when the typical size of a system becomes comparable with the mean free path of the heat carriers [39]. We assume that all ballistic transport effects were captured by the MC modeling of the high-energy electrons described previously, and the low-energy electrons only exhibit the diffusive behavior. When this is not the case, a more general approach should be used, such as the ballistic-diffusive equation [39], which is beyond the scope of the present paper.

The TTM is a set of two coupled nonlinear differential equations, which describe the evolution of electron $\left(T_{e}\right)$ and lattice $\left(T_{l}\right)$ temperatures as functions of depth $(z)$ and time $(t)$ induced by absorption of an ultrashort laser pulse:

$$
\left\{\begin{array}{c}
C_{e} \frac{\partial T_{e}}{\partial t}=\frac{\partial}{\partial z}\left(k_{e} \frac{\partial T_{e}}{\partial z}\right)-G\left(T_{e}-T_{l}\right)+S(z, t) \\
C_{l} \frac{\partial T_{l}}{\partial t}=G\left(T_{e}-T_{l}\right)
\end{array} .\right.
$$

$C_{e}$ and $C_{l}$ are the electron and lattice heat capacities, respectively; $k_{e}$ is the electron thermal conductivity; $\mathrm{G}$ is the electronphonon coupling factor. The lattice thermal conductivity is usually neglected for metals since it is typically much smaller than the electron thermal conductivity. $S(z, t)$ is the heat source, which is obtained from XCASCADE(3D) as the derivative of the energy density $U(z, t)$ of low-energy electrons and valence holes:

$$
S(z, t)=\frac{\partial U(z, t)}{\partial t}, \quad z \in[0, L],
$$

where $L$ is the film thickness. The problem is formulated in only one in-depth dimension $z$, since the typical laser spot size $(\sim \mu \mathrm{m}$, see Ref. [13]) is much larger than the penetration depth of the radiation $(\sim \mathrm{nm})$, which makes the temperature gradients in the lateral direction much smaller than in the in-depth direction.

The TTM assumes that the atomic lattice can be described in terms of phonons, the collective harmonic oscillators. This approximation requires that (i) the crystal has a perfect periodic structure that is undamaged during the simulation; (ii) the interatomic potential can be approximated as harmonic and does not change in time (due to, e.g., nonthermal effects); and (iii) the characteristic times of the studied processes are larger than the characteristic phonon time (inverse phonon frequency). Strictly speaking, these conditions may not be satisfied under femtosecond FEL pulse irradiation; however, more general approaches to electron-ion energy exchange are computationally much more demanding and will not be used in this work $[40,41]$.

The TTM just formulated is solved numerically using a finite difference method. The initial and boundary conditions are the following:

$$
\begin{gathered}
T_{e}\left(z,-2 \tau_{p}\right)=T_{l}\left(z,-2 \tau_{p}\right)=300 \mathrm{~K}, \quad z \in[0, L], \\
\left.\frac{\partial T_{e}}{\partial z}\right|_{z=0}=\left.\frac{\partial T_{l}}{\partial z}\right|_{z=0}=\left.\frac{\partial T_{e}}{\partial z}\right|_{z=L}=\left.\frac{\partial T_{l}}{\partial z}\right|_{z=L}=0,
\end{gathered}
$$

where $\tau_{p}$ is the pulse duration. As one can see, thermally isolated boundaries are used during the entire simulation, which corresponds to a free-standing film. For this approximation to be accurate, the thickness of the film should be large enough to make sure that the supporting substrate does not affect the thermal evolution of the film near the irradiated boundary.

The enthalpy approach [42] is used in order to take melting into account. The difference in thermal properties between the liquid and the solid phase are neglected for simplicity. The lattice heat capacity dependence on the lattice temperature is extracted from the relationship between the enthalpy and the lattice temperature taken from Ref. [43].

The results of the TTM calculations strongly depend on the choice of the thermal parameters for a particular material of 
interest. Generally, all parameters are temperature dependent. In the regime of high fluences, the electron temperature can reach high values on the order of the Fermi temperature or higher. In such a regime, thermal parameters may differ significantly from their room temperature values. In this work, we study various approximations for the model parameters of $\mathrm{Ru}$, as well as their applicability and influence on the behavior of electron and lattice temperatures, with the latter being crucial for understanding the mechanisms of laser-induced damage in metals.

\section{RESULTS AND DISCUSSION}

\section{A. Model Parameters of Ru}

In this section, we describe the different approximations for the following model parameters of $\mathrm{Ru}$ : photoelectron velocity distribution (due to the photon polarization), electron cutoff energy (separating high-energy from low-energy electrons in the MC scheme), electron heat capacity, electron thermal conductivity, and electron-phonon coupling factor.

\section{Photoelectron Velocity Distribution}

In a single-shot damage experiment reported in Ref. [13], the authors used p-polarized light with respect to the sample surface. Generally, polarization influences the direction of photoelectrons emitted after absorption of the photons. In order to study the effect of polarization on the final energy and density distributions of thermalized electrons and valence holes, we consider two limiting cases. In the first case, we assume random directions of photoelectrons (isotropic), as if the polarization had no effect. In the other limiting case, we allow photoelectrons to travel only perpendicular to the surface (up and down), mimicking p-polarization of a pulse under grazing incidence conditions. In that case, the effect of polarization is most pronounced: more electrons and energy are expected to be emitted from the surface on the one hand, and propagate deeper into $\mathrm{Ru}$ on the other hand.

The comparison between these two limiting cases is shown in Fig. 2. The total density profiles of thermalized electrons
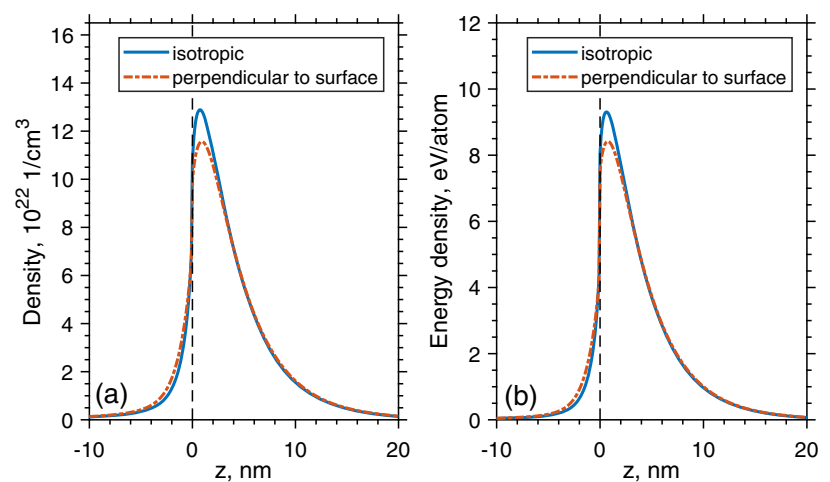

Fig. 2. Depth profiles of total (a) number density and (b) energy density of thermalized electrons and valence holes at $t=200 \mathrm{fs}$, calculated with the XCASCADE(3D) code for an incident fluence $F=200 \mathrm{~mJ} / \mathrm{cm}^{2}$. Solid lines are the results of calculations with isotropic directions of photoelectrons, while dash-dotted lines are calculation results with directions of photoelectrons perpendicular to the surface. The cutoff energy is $6.38 \mathrm{eV}$. Vertical dashed lines mark the surface of $\mathrm{Ru}$, with the $z<0$ region treated as vacuum. (those with energy below the cutoff of $6.38 \mathrm{eV}$ ) and valence holes at time $t=200 \mathrm{fs}$ are plotted in Fig. 2(a). Figure 2(b) shows the total energy density. The results demonstrate only minor differences: the peaks are reduced by about $10 \%$ for the polarized case, while the tails of the distributions practically coincide. The time when all electron cascades are already finished is chosen as $200 \mathrm{fs}$.

We conclude that at the photon energy of $92 \mathrm{eV}$, the polarization does not affect the electronic transport significantly. We expect, however, that the effects will be more pronounced for higher photon energies. For all further calculations in this work, we choose the initial velocities of the photoelectrons to be perpendicular to the surface, since this case is closer to the experimental conditions used in Ref. [13]

\section{Cutoff Energy of Cascading Electrons}

We perform a similar comparison to that just described, but vary the energy cutoff in the MC scheme, which separates the cascading electrons from the thermalized ones. Two values are considered: $10 \mathrm{eV}$, which is a standard cutoff energy used in previous studies of electron cascading [25,31], and $6.38 \mathrm{eV}$, which corresponds to the ionization potential of the outermost shell (5s) of the Ru atom [44]. By the ionization potential, we mean the energy necessary to promote an electron from the $5 \mathrm{~s}$ state into the unoccupied delocalized states of the conduction band. In the case of $6.38 \mathrm{eV}$ cutoff energy, electrons have additional freedom for the final impact ionization of the $5 \mathrm{~s}$ shell before their motion is stopped and they are considered as thermalized. The comparison is presented in Fig. 3.

The total density, Fig. 3(a), is slightly higher in the case of $6.38 \mathrm{eV}$ cutoff energy, since an additional impact ionization event of the $5 \mathrm{~s}$ shell leads to a higher number of thermalized electrons and valence $5 \mathrm{~s}$ holes at the end of the cascading process. The energy density, Fig. 3(b), in that case is slightly more spread in space, since a lower cutoff energy allows the electrons to travel slightly further. However, the total energy of the system is the same for both cases.
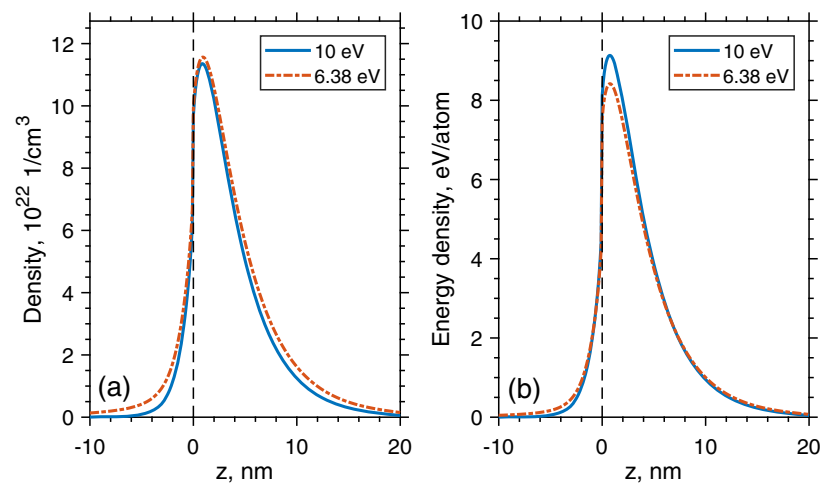

Fig. 3. Depth profiles of total (a) number density and (b) energy density of thermalized electrons and valence holes at $t=200 \mathrm{fs}$, calculated with the XCASCADE(3D) code for an incident fluence $F=200 \mathrm{~mJ} / \mathrm{cm}^{2}$. Solid lines are the results of calculations with $10 \mathrm{eV}$ cutoff energy; dash-dotted lines are results with $6.38 \mathrm{eV}$. The velocities of photoelectrons are perpendicular to the surface. Vertical dashed lines mark the surface of $\mathrm{Ru}$, with the $z<0$ region treated as vacuum. 
Our results show that the choice of the cutoff energy (within a few $\mathrm{eV}$ margin) hardly affects the spatial energy distribution or the duration of electron cascading (not shown). Indeed, the number of escaping electrons and the amount of energy they carry away are only changed by $4 \%$ and $2 \%$, respectively. The electronic number and energy densities never differ by more than $9 \%$ for the two considered cases. In all further calculations, $6.38 \mathrm{eV}$ cutoff energy is used.

\section{Electron Heat Capacity}

The linear approximation for the electron heat capacity derived within the Sommerfeld model (free electron gas approximation) is typically used for metals in the regime of relatively low electron temperatures: $C_{e}\left(T_{e}\right)=\gamma T_{e}$, where $\gamma$ is the electron specific heat constant. In order to go beyond the free electron gas approximation and take into account a realistic density of states (DOS) of a material, we perform calculations of the electron heat capacity dependence on the electron temperature, using the formalism described in [45]. The Ru DOS is taken from Ref. [46]. The results are shown in Fig. 4(a). Dashed and solid lines are the electron heat capacity calculated with the free electron gas approximation and the DOS of $\mathrm{Ru}$, respectively. The results are close to each other up to $\sim 6000 \mathrm{~K}$, after which the increase of $C_{e}$ calculated with DOS becomes more gradual compared to the free electron gas approximation.

\section{Electron-Phonon Coupling Factor}

The temperature dependence of the electron-phonon coupling factor calculated using the formalism described in Refs. $[45,48]$ is shown in Fig. 4(b). The strong enhancement is followed by a decrease, with a maximum value reached at $T_{e} \sim 1.2 * 10^{4} \mathrm{~K}$. A similar behavior of $G$ with increasing electron temperature was previously reported for titanium [6]. Both metals have an hcp crystal structure and relatively low DOS at the Fermi level, which may explain the similar behavior. Note that at low temperatures the calculated values agree reasonably well with the experimental data from Ref. [47] [marked in Fig. 4(b) with a dashed line].
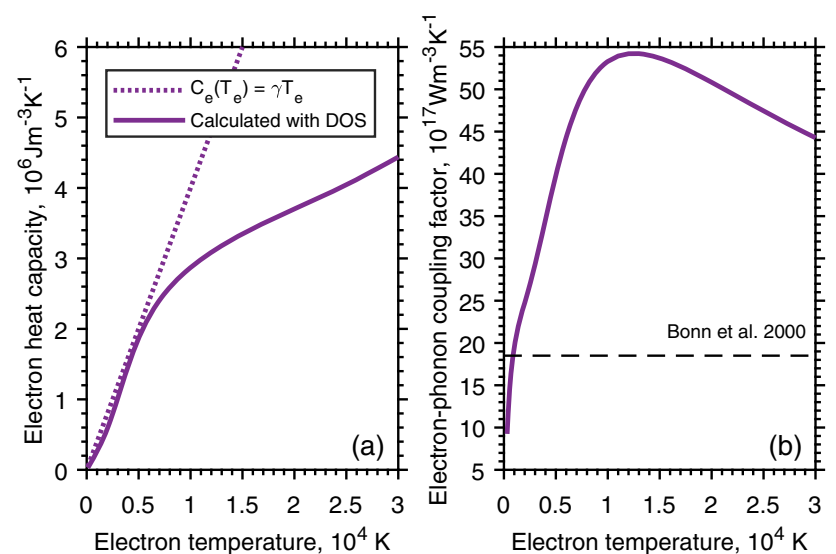

Fig. 4. Electron temperature dependence of the (a) electron heat capacity and (b) electron-phonon coupling factor calculated with the DOS of Ru taken into account (solid lines). The linear approximation for the electron heat capacity is shown with the dotted line in (a). The constant value of the electron-phonon coupling factor determined experimentally in Ref. [47] is shown with dashed lines in (b).

\section{Electron Thermal Conductivity}

Different approximations for the electron thermal conductivity $k_{e}$ are available in the literature [49]. In the regime of low electron temperatures, the following approximation is typically used:

$$
k_{e}^{\text {linear }}\left(T_{e}, T_{l}\right)=k_{0} \frac{T_{e}}{T_{l}},
$$

where $k_{0}$ is the room temperature equilibrium thermal conductivity. It is assumed here that electron-phonon scattering is the dominant scattering process. For higher electron temperatures, both electron-electron and electron-phonon scattering processes play a role, and a more general approximation for $k_{e}$ must be used [49]:

$$
k_{e}^{A, B}\left(T_{e}, T_{l}\right)=\frac{1}{3} v_{F} \gamma \frac{T_{e}}{A T_{e}^{2}+B T_{l}},
$$

where $v_{F}$ is the Fermi velocity and $A$ and $B$ are material dependent constants which are determined by the electronelectron and electron-phonon collision frequencies, respectively. Equation (6) is only valid for electron temperatures considerably smaller than the Fermi temperature; otherwise, a more general expression should be used [49,50]:

$$
k_{e}^{K, b}\left(T_{e}, T_{l}\right)=K \cdot \frac{\left(\vartheta^{2}+0.16\right)^{5 / 4}\left(\vartheta^{2}+0.44\right) \vartheta}{\left(\vartheta^{2}+0.092\right)^{1 / 2}\left(\vartheta^{2}+b \vartheta_{l}\right)} .
$$

Here, $\vartheta=k_{B} T_{e} / E_{F}$ and $\vartheta_{l}=k_{B} T_{l} / E_{F}$, where $k_{B}$ is the Boltzmann constant and $E_{F}$ is the Fermi energy. $K$ and $b$ are material dependent constants.

Although the approximations just described are known and widely applied, the constants $A$ and $B$ or $K$ and $b$ are unknown for most materials. We propose a way of determining the corresponding constants based on a measured temperature dependence of the thermal conductivity, taken from Ref. [51]. Equations (6) and (7) can be fitted to the experimental data with $A$ and $B$, or $K$ and $b$, as fitting parameters, taking $T_{e}=T_{l}=T$, since experimental values of the thermal conductivity are measured in the regime of thermal equilibrium between electrons and the lattice. Following this procedure for $\mathrm{Ru}$, we find that $A=7.82 * 10^{8} \mathrm{~s}^{-1} \mathrm{~K}^{-2}, B=$ $3.24 * 10^{12} \mathrm{~s}^{-1} \mathrm{~K}^{-1}$ [for Eq. (6)], $K=34.98 \mathrm{Wm}^{-1} \mathrm{~K}^{-1}$, and $b=0.0416$ [for Eq. (7)]. In the fitting procedure, the data from Ref. [51] are taken only up to the melting point of $\mathrm{Ru}$, which means that the influence of melting on the thermal conductivity coefficient is not taken into account.

The three approximations for electron thermal conductivity are compared in Fig. 5. The dependence on electron temperature is shown with the lattice temperature fixed at room temperature, $T_{l}=300 \mathrm{~K}$. The first approximation [Eq. (5)], which we will refer to as the linear approximation, results in a rapid increase of the electron thermal conductivity. The two other approximations [Eqs. (6) and (7)] exhibit a qualitatively different behavior. The initial increase up to $\sim 1100 \mathrm{~K}$ is followed by a significant drop of $k_{e}$. Equation (6) tends to zero with further increasing electron temperature, while Eq. (7) exhibits a second sharp increase for $T_{e} \geq 36000 \mathrm{~K}$, as expected in the plasma limit [49]. 


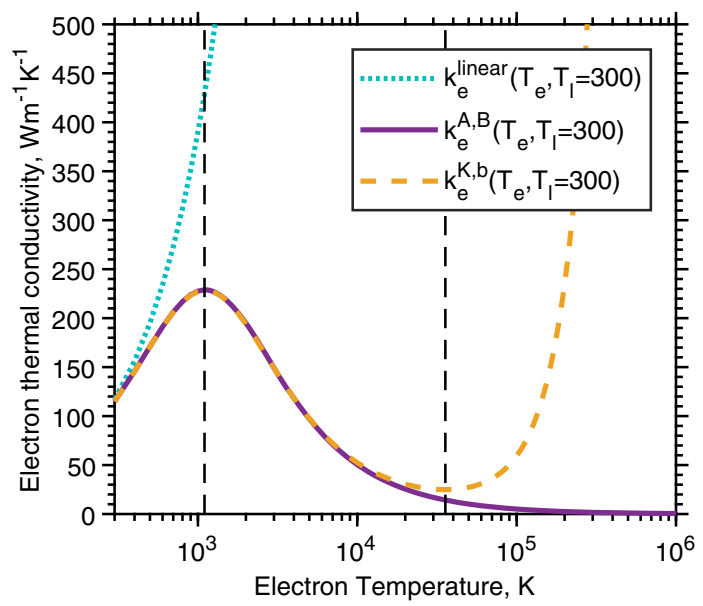

Fig. 5. Electron temperature dependence of the electron thermal conductivity in three different approximations [Eqs. (5)-(7)] plotted for a fixed lattice temperature $T_{l}=300 \mathrm{~K}$. Dashed vertical lines schematically divide the entire electron temperature range into three characteristic regions.

Based on this behavior, the electron temperature range can be approximately divided into three characteristic regions: (i) "low" temperatures, $T_{e}<1100 \mathrm{~K}$; (ii) "intermediate" temperatures, $1100 \leq T_{e} \leq 36000 \mathrm{~K}$; and (iii) "high" temperatures, $T_{e}>36000 \mathrm{~K}$. The vertical dashed lines in Fig. 5 mark these three regions. Although $k_{e}$ in all three approximations is qualitatively similar in region (i), a significant quantitative difference is reached with increasing $T_{e}$ (almost a factor of 2 difference at $T_{e}=1100 \mathrm{~K}$ ). This fact makes the linear approximation questionable to use for all temperature ranges.

The results for Eqs. (6) and (7) almost coincide in regions (i) and (ii), but differ strongly in region (iii). From this analysis we conclude that Eq. (6) is valid in regions (i) and (ii), while the most general approximation, Eq. (7), should be valid for all electron temperatures considered here.

\section{B. Influence of Thermal Parameters on Damage Characteristics}

In our recent study [13], we showed that the nature of singleshot damage of a $50 \mathrm{~nm} \mathrm{Ru}$ film induced by a $100 \mathrm{fs}$ XUV $(92 \mathrm{eV})$ FEL pulse is photomechanical spallation in the stress confinement regime. The phenomenon of spallation was extensively studied both experimentally and theoretically in the field of interaction of optical and XUV lasers with matter [14-23]. The mechanism behind laser-induced spallation in metals is as follows. The laser energy is first absorbed by the electrons in the near surface layer of a metal. Then, the excited electrons propagate into the depth of the target, simultaneously heating the lattice due to electron-phonon interaction. If the heating of the lattice by the hot electrons occurs faster than mechanical relaxation of the system, the heating is almost isochoric. As a result, large compressive stresses are generated. The compressive component of a stress wave is followed by a tensile component due to the existence of a free surface. The amplitude of a tensile stress propagating into the depth of the material is increasing until the threshold value is reached at some depth, which leads to spallation.

The condition of the stress-confinement regime can be formulated in the following way $[14,15,19]: \tau_{\mathrm{el}-\mathrm{ph}} \leq \tau_{a}$, where $\tau_{\mathrm{el}-\mathrm{ph}}$ is the electron-phonon thermalization time and $\tau_{a}$ is the acoustic relaxation time. The latter can be calculated as $\tau_{a}=L_{c} / C_{s}$, where $L_{c}$ is the electron diffusion length and $C_{s}$ is bulk speed of sound. Therefore, one needs to know the characteristic thermal time $\left(\tau_{\mathrm{el}-\mathrm{ph}}\right)$ and length $\left(L_{c}\right)$ scales of the problem in order to find out whether the regime of stress confinement is realized.

Another key process playing a role in the single-shot damage mechanism is melting. It has been shown that for metals, melting typically occurs before the spallation, so that spallation starts in a liquid material $[15,19]$. The calculated depth of melting can be compared with the experimentally observed depth of the damaged crater in order to check whether the latter is smaller than the former.

In this section, we study the influence of the particular choice of thermal parameters of Ru on the electron and lattice temperature behavior, and on the characteristic values playing a role in the damage process, such as the electron-phonon thermalization time, electron diffusion length, and melted depth. The analysis is performed in such a way that each parameter $\left(C_{e}, G\right.$, and $\left.k_{e}\right)$ is varied, fixing the choice of the approximations for the other two.

Combined XCASCADE(3D) and TTM calculations are performed in the same way as described previously, and (in more detail) in Ref. [13]. All parameters used in the simulations are for bulk $\mathrm{Ru}$, and are summarized in Table 1 . The incident fluence level used in all the simulations is chosen to be $F=200 \mathrm{~mJ} / \mathrm{cm}^{2}$, which is the experimentally determined

Table 1. Model Parameters

\begin{tabular}{|c|c|}
\hline Film thickness & $L=200 \mathrm{~nm}$ \\
\hline Photon energy & $92 \mathrm{eV}$ \\
\hline Grazing incidence angle & $20^{\circ}$ \\
\hline Fermi energy $^{a}$ & $E_{F}=8.5 \mathrm{eV}$ \\
\hline Pulse duration & $\tau_{p}=100 \mathrm{fs}$ \\
\hline Surface reflectivity ${ }^{b}$ & $R=0.68$ \\
\hline Electron emission coefficient ${ }^{c}$ & $\alpha=0.12$ \\
\hline Incident fluence & $F=200 \mathrm{~mJ} / \mathrm{cm}^{2}$ \\
\hline Absorbed fluence & $F_{\mathrm{abs}}=56 \mathrm{~mJ} / \mathrm{cm}^{2}$ \\
\hline Photon attenuation length ${ }^{b}$ & $\delta=3.4 \mathrm{~nm}$ \\
\hline Electron-specific heat constant ${ }^{d}$ & $\gamma=400 \mathrm{~J} / \mathrm{m}^{3} / \mathrm{K}^{2}$ \\
\hline $\begin{array}{l}\text { Electron-phonon coupling } \\
\text { factor }^{e}\end{array}$ & $G^{\mathrm{const}}=18.5 * 10^{17} \mathrm{~W} / \mathrm{m}^{3} / \mathrm{K}$ \\
\hline $\begin{array}{l}\text { Equilibrium thermal } \\
\text { conductivity }\end{array}$ & $k_{0}=117 \mathrm{~W} / \mathrm{m} / \mathrm{K}$ \\
\hline Latent heat of melting ${ }^{g}$ & $H_{m}=4.7 * 10^{9} \mathrm{~J} / \mathrm{m}^{3}$ \\
\hline Bulk modulus $^{h}$ & $B=310 \mathrm{GPa}$ \\
\hline Density ${ }^{g}$ & $\rho=12.3 \mathrm{~g} / \mathrm{cm}^{3}$ \\
\hline
\end{tabular}

${ }^{a}$ Ref. [46].

${ }^{b}$ Ref. [29], at $92 \mathrm{eV}$ photon energy, $20^{\circ}$ grazing incidence.

'Extracted from Fig. 2(b).

${ }^{d}$ Ref. [52].

${ }^{e}$ Ref. [47].

${ }^{\text {Ref. [51]. }}$

${ }^{8}$ Ref. [43].

${ }^{b}$ Ref. [53]. 
spallation threshold of $\mathrm{Ru}$ [13]. The corresponding absorbed fluence is calculated as $F_{\mathrm{abs}}=F(1-R)(1-\alpha)$, where $R$ is surface reflectivity and $\alpha$ is the fraction of the energy that escapes from the surface due to electron emission. The latter is estimated to be $\sim 12 \%$ for the case of $6.38 \mathrm{eV}$ cutoff energy and velocities of photoelectrons perpendicular to the surface [extracted from Fig. 2(b)]. The thickness of the film $L$ is taken to be $200 \mathrm{~nm}$. In that way, we make sure that the increase of the rear surface temperature of the film is negligible compared to the changes of the front surface temperatures, so that the $\mathrm{Si}$ substrate can be excluded from the calculations.

\section{Influence of Parameters on Electron and Lattice Temperatures}

Figure 6(a) compares the temporal evolution of electron and lattice surface temperatures for different electron heat capacities. Calculations with $C_{e}^{\mathrm{DOS}}$ result into a much higher electron temperature peak value, since $C_{e}^{\mathrm{DOS}}$ is significantly lower than $C_{e}^{\text {linear }}$ at electron temperatures on the order of $2-3 * 10^{4} \mathrm{~K}$ [see Fig. 4(a)]. Although the initial difference in temperatures (both electron and lattice) between calculations with $C_{e}^{\text {linear }}$ and $C_{e}^{\mathrm{DOS}}$ is noticeable, the strong electronphonon coupling factor of $\mathrm{Ru}$ [see Fig. 4(b)] results in rapid attainment of thermal equilibrium between electrons and the lattice, after which the difference in temperatures is negligible. This is confirmed with electron temperature depth profiles plotted at different moments of time, shown in Fig. 6(b). The small difference in temperatures in the near surface region before electron-phonon thermalization $(t=0.5 \mathrm{ps})$ vanishes at later times. The fact that $k_{e}$ and $G$ dependencies are fixed results in almost identical depth profiles for the entire thickness of the film. Note that here and further only the top $50 \mathrm{~nm}$ of the total $200 \mathrm{~nm}$ thickness is shown.

A similar analysis is performed for two other thermal parameters: $G$ and $k_{e}$. Figure 7 compares calculations performed for a constant value of $G$ (measured at room temperature, see Ref. [47]) and for electron temperature dependent $G\left(T_{e}\right)$ obtained with the DOS taken into account [Fig. 4(b)]. In contrast to the variation of $C_{e}$ described previously, different $G$
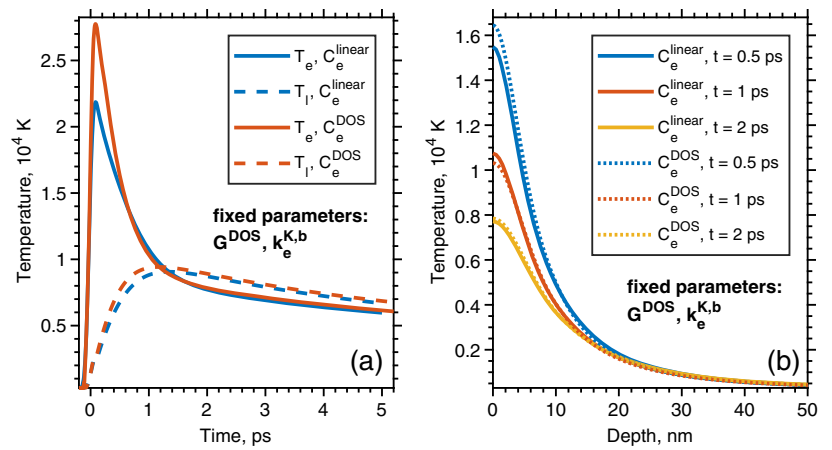

Fig. 6. (a) Calculated temporal evolution of electron $\left(T_{e}\right.$, solid lines) and lattice ( $T_{l}$, dashed lines) surface temperatures of a $200 \mathrm{~nm}$ $\mathrm{Ru}$ film irradiated by a $100 \mathrm{fs}$ XUV pulse. Absorbed fluence is $F_{\text {abs }}=56 \mathrm{~mJ} / \mathrm{cm}^{2}$. Electron heat capacity $C_{e}$ is a varied parameter, and electron-phonon coupling factor and electron thermal conductivity are fixed at $G^{\mathrm{DOS}}, k_{e}^{K, b}$ values. (b) Electron temperature depth profiles at different times calculated under the same conditions.
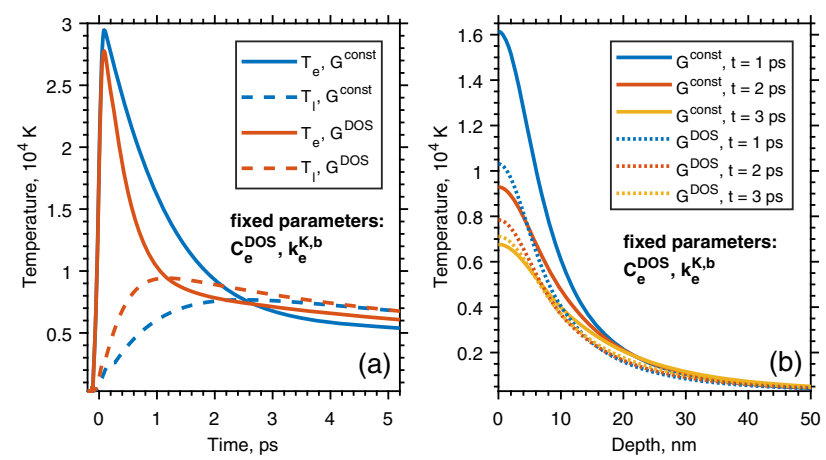

Fig. 7. Same as Fig. 6 but for electron-phonon coupling factor as a varied parameter, and electron heat capacity and electron thermal conductivity fixed at $C_{e}^{\mathrm{DOS}}, k_{e}^{K, b}$ values.

functions almost do not affect the electron temperature peak value, while the dynamics of electron-phonon thermalization is different, as could be expected [Fig. 7(a)]. Stronger electronphonon coupling $\left[G^{\mathrm{DOS}}\right.$, see Fig. 4(b)] results in earlier thermal equilibrium compared to a constant value of $G$. Electron temperature depth profiles, Fig. 7(b), demonstrate a significant difference in temperature in the top $\sim 20 \mathrm{~nm}$ of $\mathrm{Ru}$ at early times ( $t=1,2 \mathrm{ps}$ ), although the difference decreases with time $(t=3 \mathrm{ps})$. No significant difference in temperatures profiles is found for deeper parts of Ru.

Figure 8 compares the calculations performed with different approximations for the electron thermal conductivity $k_{e}$. There is almost no difference in temperature behavior between calculations with $k_{e}^{A, B}$ [Eq. (6)] and $k_{e}^{K, b}$ [Eq. (7)], which is not surprising, since these approximations are almost identical for the electron temperature range in the simulations (see Fig. 5). However, a larger difference is expected for higher fluences. A dramatic difference is observed when comparing $k_{e}^{A, B}$ or $k_{e}^{K, b}$ with the linear approximation. A much higher $k_{e}^{\text {linear }}$ leads to much faster heat transport from the surface into the depth of the $\mathrm{Ru}$ film. As a result, the model gives significantly lower surface temperatures [Fig. 8(a)]. The electron thermal conductivity significantly affects the distribution of the absorbed energy in the $\mathrm{Ru}$ film. Lower $k_{e}$ in Eqs. (6) and (7) result in confinement of heat in the top $\sim 20 \mathrm{~nm}$ during the first few ps after the pulse, while for a higher $k_{e}$ in the linear
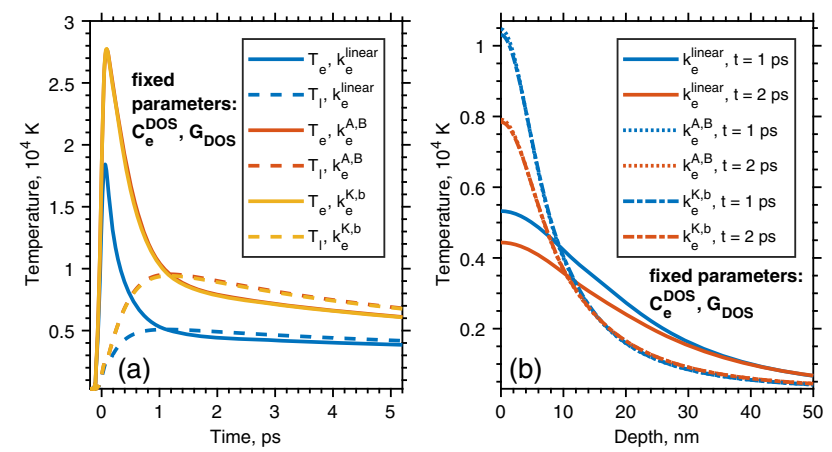

Fig. 8. Same as Fig. 6 but for electron thermal conductivity as a varied parameter, and electron heat capacity and electron-phonon coupling factor fixed at $C_{e}^{\mathrm{DOS}}, G^{\mathrm{DOS}}$ values. 

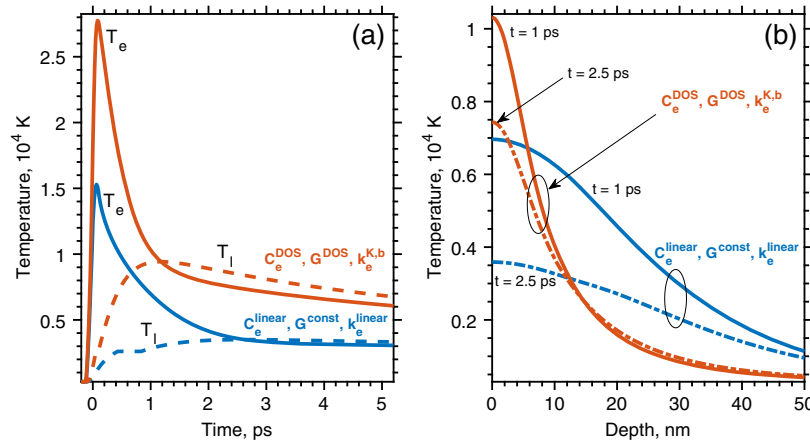

Fig. 9. Same as Fig. 6 but with the following sets of thermal parameters compared: $\left(C_{e}^{\text {linear }}, G^{\text {const }}, k_{e}^{\text {linear }}\right)$ and $\left(C_{e}^{\mathrm{DOS}}, G^{\mathrm{DOS}}, k_{e}^{K, b}\right)$.

approximation, the heat propagates much deeper. This indicates that the simple linear approximation may result in significant underestimation of the temperatures.

Summarizing the analysis performed, we found that the electron thermal conductivity has the most significant impact on electron and lattice temperature evolution in irradiated $\mathrm{Ru}$.

Finally, to emphasize the importance of a proper choice of thermal parameters, we compare calculations of temperature evolution for two sets of parameters: the simplest set $\left(C_{e}^{\text {linear }}\right.$, $G^{\text {const }}, k_{e}^{\text {linear }}$ ) and (presumably) the most accurate one $\left(C_{e}^{\mathrm{DOS}}, G^{\mathrm{DOS}}, k_{e}^{K, b}\right)$. The results are shown in Fig. 9, illustrating the dramatic qualitative and quantitative difference in temperature behavior.

\section{Influence of Parameters on the Melting Dynamics}

As described previously, melting plays an important role in laser-induced damage of metals. We demonstrate the influence of thermal parameters on the melting dynamics in the example of varying electron thermal conductivity, since we showed that this parameter has the strongest impact on the temperature behavior. No significant difference was found between Eqs. (6) and (7) for the temperature range obtained in the simulations, and therefore we will focus on comparing the linear approximation with the most accurate one, Eq. (7). The other two thermal parameters are chosen as those with the DOS taken into account $\left(C_{e}^{\mathrm{DOS}}, G^{\mathrm{DOS}}\right)$.

Figure 10 shows such a comparison for the calculated depth of melting changing with time. Both curves exhibit a similar behavior: (i) fast melting of the top 10-15 $\mathrm{nm}$ of Ru during the first 2 ps in the regime of thermal non-equilibrium between electrons and lattice, (ii) slower propagation of the melting front in the equilibrium regime before the maximum depth of melting is reached, and (iii) cooling down and recrystallization. Although the general behavior is similar, the dynamics of melting and recrystallization is different. Higher thermal conductivity in the linear approximation makes the heat diffusion from the surface into the depth of the material much faster. As a result, melting starts slightly later, but propagates faster.

The maximum depth of melting $L_{\text {melt }}$ is almost the same for the two approximations $\left(L_{\text {melt }}=16.5\right.$ and $15.8 \mathrm{~nm}$ for $k_{e}^{\text {linear }}$ and $k_{e}^{K, b}$, respectively), but is reached at different times: at $\sim 13$ ps for $k_{e}^{\text {linear }}$ and at $\sim 27$ ps for $k_{e}^{K, b}$. Both values of $L_{\text {melt }}$ are larger than or equal to the experimentally determined

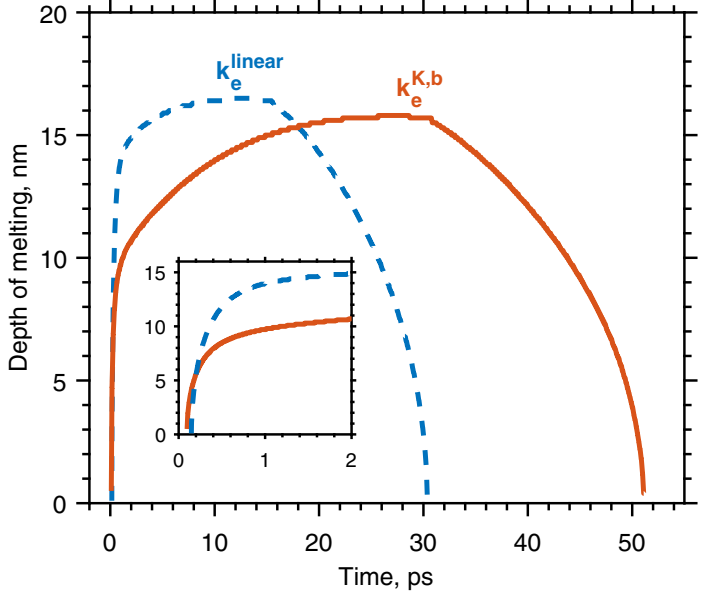

Fig. 10. Calculated transient depth of melting in $200 \mathrm{~nm} \mathrm{Ru}$ film irradiated by a $100 \mathrm{fs}$ XUV pulse. The absorbed fluence is $F_{\text {abs }}=56 \mathrm{~mJ} / \mathrm{cm}^{2}$. Calculations with two approximations for the electron thermal conductivity are compared: $k_{e}^{\text {linear }}$ (dashed line) and $k_{e}^{K, b}$ (solid line). The initial stage of melting is shown in the inset.

thickness of the spallated layer at the spallation threshold (5-16 nm, see Ref. [13]), which is consistent with the assumption that spallation starts in a melted material.

The same value of $L_{\text {melt }}$ for different $k_{e}$ approximations can be explained by the fact that the total amount of melted material after irradiation with a femtosecond pulse is mostly determined by the absorbed fluence, heat capacity, and latent heat of melting $H_{m}$ as $L_{\text {melt }} \sim F_{\text {abs }} /\left(C_{l}\left(T_{\text {melt }}-T_{0}\right)+H_{m}\right)$. The thermal conductivity only has a strong effect on how fast $L_{\text {melt }}$ is reached. The slightly larger value of $L_{\text {melt }}$ in the case of calculations with $k_{e}^{\text {linear }}$ is due to the fact that, for a higher thermal conductivity, a larger amount of energy diffuses away from the melted region before $L_{\text {melt }}$ is reached. Cooling and ensuing recrystallization is also faster for a higher $k_{e}^{\text {linear }}$

\section{Influence of Parameters on the Stress Confinement Condition}

To check whether the condition of stress confinement is satisfied, one needs to know the thermal and mechanical characteristic time scales $\tau_{\mathrm{el}-\mathrm{ph}}$ and $\tau_{a}$, respectively. The electron-phonon thermalization time $\tau_{\mathrm{el}-\mathrm{ph}}$ is defined as the time when the normalized difference between the electron and lattice surface temperatures decreases to the $1 / e$ level. To determine $\tau_{a}$, the electron diffusion length $L_{c}$ is extracted from the electron temperature depth profile at $t=\tau_{\mathrm{el}-\mathrm{ph}}$, as the depth where the normalized temperature decreases to the $1 / e$ level. The bulk speed of sound $C_{s}$ is calculated as $C_{s}=\sqrt{B / \rho} \sim 5000 \mathrm{~m} / \mathrm{sec}$, where $B$ is the bulk modulus and $\rho$ is the density.

We found that the condition of stress confinement is satisfied for all possible parameter combinations, although the particular values of $\tau_{\mathrm{el}-\mathrm{ph}}$ and $L_{c}$ vary significantly. This is illustrated in Table 2, where $\tau_{\mathrm{el}-\mathrm{ph}}$ and $L_{c}$ (and corresponding $\left.\tau_{a}\right)$, together with the maximum values of the electron and lattice temperatures, are shown for two sets of thermal parameters, namely $\left(C_{e}^{\text {linear }}, G^{\text {const }}, k_{e}^{\text {linear }}\right)$ and $\left(C_{e}^{\mathrm{DOS}}, G^{\mathrm{DOS}}, k_{e}^{K, b}\right)$, the same as in Fig. 9. 
Table 2. Calculated Thermal and Mechanical Characteristics of Ru Irradiated by a 100 fs XUV Laser Pulse $^{a}$

\begin{tabular}{lcc}
\hline & $C_{\boldsymbol{e}}^{\text {linear }}, \boldsymbol{G}^{\text {const }}, \boldsymbol{k}_{\boldsymbol{e}}^{\text {linear }}$ & $\boldsymbol{C}_{\boldsymbol{e}}^{\mathrm{DOS}}, \boldsymbol{G}^{\mathrm{DOS}}, \boldsymbol{k}_{\boldsymbol{e}}^{\boldsymbol{K}, \boldsymbol{b}}$ \\
\hline$\tau_{\mathrm{el}-\mathrm{ph}}[\mathrm{ps}]$ & 0.82 & 0.48 \\
$L_{c}[\mathrm{~nm}]$ & 38 & 11 \\
$\tau_{a}[\mathrm{ps}]$ & 7.6 & 2.2 \\
$T_{e}^{\max }\left[10^{4} \mathrm{~K}\right]$ & 1.53 & 2.78 \\
$T_{l}^{\max }\left[10^{4} \mathrm{~K}\right]$ & 0.35 & 0.94 \\
\hline
\end{tabular}

${ }^{a}$ Absorbed fluence is $F_{\text {abs }}=56 \mathrm{~mJ} / \mathrm{cm}^{2}$.

As one can see from Table 2 and Fig. 9, different $k_{e}$ values strongly affect the electron diffusion length $L_{c}$ and, as a result, the maximum values of both the electron and lattice temperatures. In the case of irradiation of a Ru film with a femtosecond XUV laser pulse considered in this work, the stressconfinement regime is satisfied for any set of available thermal parameters. Hence, the mechanism of damage does not depend on the particular choice. However, the quantitative description of the processes differs significantly. Moreover, for other materials or laser pulse parameters, inaccurate choice of the thermal parameters may lead to unreliable conclusions about the nature of laser-induced damage and its kinetic pathways. Dedicated experimental studies are required to validate thermal parameters in the regime of high electron temperatures reached during the laser ablation of metals.

\section{CONCLUSIONS}

We performed the analysis of model parameters used in simulations of interaction of a high-fluence femtosecond XUV FEL pulse with a Ru target. For simulations, we used a combined approach where photoabsorption and non-equilibrium electron kinetics were modeled with the Monte Carlo code $\mathrm{XCASCADE}(3 \mathrm{D})$, and the electron and lattice temperature evolution was described with the TTM. Variation of the parameters used in the XCASCADE(3D) part of the simulations (photoelectron velocity distribution and energy cutoff) showed no significant difference in the description of electron cascades, although larger differences are expected for higher photon energies.

The following thermal parameters were varied within the TTM part of the simulations: electron heat capacity, electronphonon coupling factor, and electron thermal conductivity. The latter was found to have a major impact on temperature behavior and, hence, on the description of single-shot damage processes. Although we found that the condition of stress confinement, proposed as the key mechanism responsible for damage, is fulfilled for all possible parameter combinations in our particular case, it may not hold universally for other materials or laser parameters. Moreover, the choice of model parameters considerably affects the temporal kinetics of heating and relaxation of the target. Therefore, the proper choice of model parameters, especially the electronic thermal conductivity in the regime of high electron temperatures, is important.
Funding. The Dutch Topconsortia Kennis en Innovatie (TKI) Program on High-Tech Systems and Materials (14 HTSM 05); Czech Ministry of Education (LTT17015, LM2015083).

Acknowledgment. The authors acknowledge support from the Industrial Focus Group XUV Optics of the MESA+ Institute for Nanotechnology of the University of Twente; industrial partners ASML, Carl Zeiss SMT GmbH, and Malvern Panalytical, the Province of Overijssel, and the Netherlands Organisation for Scientific Research (NWO). The authors acknowledge helpful discussions with Beata Ziaja (Center for Free-Electron Laser Science, Deutsches Elektronen-Synchrotron DESY) as well as her contribution to the development of the XCASCADE(3D) code.

\section{REFERENCES}

1. S. Schreiber and B. Faatz, "The free-electron laser flash," in High Power Laser Science and Engineering (2015), Vol. 3, p. e20.

2. C. Bostedt, S. Boutet, D. M. Fritz, Z. Huang, H. J. Lee, H. T. Lemke, A. Robert, W. F. Schlotter, J. J. Turner, and G. J. Williams, "Linac coherent light source: the first five years," Rev. Mod. Phys. 88, 015007 (2016).

3. C. J. Milne, T. Schietinger, M. Aiba, A. Alarcon, J. Alex, A. Anghel, V. Arsov, C. Beard, P. Beaud, S. Bettoni, M. Bopp, H. Brands, M. Brönnimann, I. Brunnenkant, M. Calvi, A. Citterio, P. Craievich, M. C. Divall, M. Dällenbach, M. D’Amico, A. Dax, Y. Deng, A. Dietrich, R. Dinapoli, E. Divall, S. Dordevic, S. Ebner, C. Erny, H. Fitze, U. Flechsig, R. Follath, F. Frei, F. Gärtner, R. Ganter, T. Garvey, Z. Geng, I. Gorgisyan, C. Gough, A. Hauff, C. P. Hauri, N. Hiller, T. Humar, S. Hunziker, G. Ingold, R. Ischebeck, M. Janousch, P. Juranić, M. Jurcevic, M. Kaiser, B. Kalantari, R. Kalt, B. Keil, C. Kittel, G. Knopp, W. Koprek, H. T. Lemke, T. Lippuner, D. L. Sancho, F. Löhl, C. Lopez-Cuenca, F. Märki, F. Marcellini, G. Marinkovic, I. Martiel, R. Menzel, A. Mozzanica, K. Nass, G. L. Orlandi, C. O. Loch, E. Panepucci, M. Paraliev, B. Patterson, B. Pedrini, M. Pedrozzi, P. Pollet, C. Pradervand, E. Prat, P. Radi, J.-Y. Raguin, S. Redford, J. Rehanek, J. Réhault, S. Reiche, M. Ringele, J. Rittmann, L. Rivkin, A. Romann, M. Ruat, C. Ruder, L. Sala, L. Schebacher, T. Schilcher, V. Schlott, T. Schmidt, B. Schmitt, X. Shi, M. Stadler, L. Stingelin, W. Sturzenegger, J. Szlachetko, D. Thattil, D. M. Treyer, A. Trisorio, W. Tron, S. Vetter, C. Vicario, D. Voulot, M. Wang, T. Zamofing, C. Zellweger, R. Zennaro, E. Zimoch, R. Abela, L. Patthey, and H.-H. Braun, "Swissfel: the Swiss X-ray Free Electron Laser," Appl. Sci. 7, 720777 (2017).

4. D. Pile, "X-rays: first light from SACLA," Nat. Photonics 5, 456-457 (2011).

5. E. Allaria, C. Callegari, D. Cocco, W. M. Fawley, M. Kiskinova, C. Masciovecchio, and F. Parmigiani, "The fermi@elettra freeelectron-laser source for coherent x-ray physics: photon properties, beam transport system and applications," New J. Phys. 12, 075002 (2010).

6. Z. Lin, L. V. Zhigilei, and V. Celli, "Electron-phonon coupling and electron heat capacity of metals under conditions of strong electron-phonon nonequilibrium," Phys. Rev. B 77, 075133 (2008).

7. X. Wang, D. M. Riffe, Y.-S. Lee, and M. Downer, "Time-resolved electron-temperature measurement in a highly excited gold target using femtosecond thermionic emission," Phys. Rev. B 50, 80168019 (1994).

8. W.-L. Chan, R. S. Averback, D. G. Cahill, and A. Lagoutchev, "Dynamics of femtosecond laser-induced melting of silver," Phys. Rev. B 78, 214107 (2008).

9. I. A. Makhotkin, R. Sobierajski, J. Chalupský, K. Tiedtke, G. de Vries, M. Störmer, F. Scholze, F. Siewert, R. W. E. van de Kruijs, I. Milov, 
E. Louis, I. Jacyna, M. Jurek, D. Klinger, L. Nittler, Y. Syryanyy, L. Juha, V. Hájková, V. Vozda, T. Burian, K. Saksl, B. Faatz, B. Keitel, E. Plönjes, S. Schreiber, S. Toleikis, R. Loch, M. Hermann, S. Strobel, H.-K. Nienhuys, G. Gwalt, T. Mey, and H. Enkisch, "Experimental study of EUV mirror radiation damage resistance under long-term free-electron laser exposures below the single-shot damage threshold," J. Synchrotron Radiat. 25, 77-84 (2018).

10. A. Aquila, R. Sobierajski, C. Ozkan, V. Hájková, T. Burian, J. Chalupský, L. Juha, M. Störmer, S. Bajt, M. T. Klepka, P. Dłużewski, K. Morawiec, H. Ohashi, T. Koyama, K. Tono, Y. Inubushi, M. Yabashi, H. Sinn, T. Tschentscher, A. P. Mancuso, and J. Gaudin, "Fluence thresholds for grazing incidence hard x-ray mirrors," Appl. Phys. Lett. 106, 241905 (2015).

11. T. Koyama, H. Yumoto, K. Tono, T. Sato, T. Togashi, Y. Inubushi, T. Katayama, J. Kim, S. Matsuyama, H. Mimura, M. Yabashi, K. Yamauchi, and $\mathrm{H}$. Ohashi, "Damage threshold investigation using grazing incidence irradiation by hard x-ray free electron laser," Proc. SPIE 8848, 88480T (2013).

12. O. Peyrusse, J.-M. André, P. Jonnard, and J. Gaudin, "Modeling of the interaction of an x-ray free-electron laser with large finite samples," Phys. Rev. E 96, 043205 (2017).

13. I. Milov, I. A. Makhotkin, R. Sobierajski, N. Medvedev, V. Lipp, J. Chalupský, J. M. Sturm, K. Tiedtke, G. de Vries, M. Störmer, F. Siewert, R. van de Kruijs, E. Louis, I. Jacyna, M. Jurek, L. Juha, V. Hájková, V. Vozda, T. Burian, K. Saksl, B. Faatz, B. Keitel, E. Plönjes, S. Schreiber, S. Toleikis, R. Loch, M. Hermann, S. Strobel, H.-K. Nienhuys, G. Gwalt, T. Mey, H. Enkisch, and F. Bijkerk, "Mechanism of single-shot damage of Ru thin films irradiated by femtosecond extreme UV free-electron laser," Opt. Express 26, 19665-19685 (2018).

14. E. Leveugle, D. Ivanov, and L. Zhigilei, "Photomechanical spallation of molecular and metal targets: molecular dynamics study," Appl. Phys. A 79, 1643-1655 (2004).

15. B. J. Demaske, V. V. Zhakhovsky, N. A. Inogamov, and I. I. Oleynik, "Ablation and spallation of gold films irradiated by ultrashort laser pulses," Phys. Rev. B 82, 064113 (2010).

16. J.-M. Savolainen, M. S. Christensen, and P. Balling, "Material swelling as the first step in the ablation of metals by ultrashort laser pulses," Phys. Rev. B 84, 193410 (2011).

17. S. I. Ashitkov, N. A. Inogamov, V. V. Zhakhovskii, Y. N. Emirov, M. B. Agranat, I. I. Oleinik, S. I. Anisimov, and V. E. Fortov, "Formation of nanocavities in the surface layer of an aluminum target irradiated by a femtosecond laser pulse," JETP Lett. 95, 176-181 (2012).

18. C. Wu and L. V. Zhigilei, "Microscopic mechanisms of laser spallation and ablation of metal targets from large-scale molecular dynamics simulations," Appl. Phys. A 114, 11-32 (2014).

19. L. V. Zhigilei, Z. Lin, and D. S. Ivanov, "Atomistic modeling of short pulse laser ablation of metals: connections between melting, spallation, and phase explosion," J. Phys. Chem. C 113, 11892-11906 (2009).

20. A. Y. Faenov, N. A. Inogamov, V. V. Zhakhovskii, V. A. Khokhlov, K. Nishihara, Y. Kato, M. Tanaka, T. A. Pikuz, M. Kishimoto, M. Ishino, M. Nishikino, T. Nakamura, Y. Fukuda, S. V. Bulanov, and T. Kawachi, "Low-threshold ablation of dielectrics irradiated by picosecond soft x-ray laser pulses," Appl. Phys. Lett. 94, 231107 (2009).

21. M. Ishino, A. Y. Faenov, M. Tanaka, N. Hasegawa, M. Nishikino, S. Tamotsu, T. A. Pikuz, N. A. Inogamov, V. V. Zhakhovsky, I. Y. Skobelev, V. E. Fortov, V. A. Khohlov, V. V. Shepelev, T. Ohba, T. Kaihori, Y. Ochi, T. Imazono, and T. Kawachi, "Nanoscale surface modifications and formation of conical structures at aluminum surface induced by single shot exposure of soft x-ray laser pulse," J. Appl. Phys. 109, 013504 (2011).

22. G. Norman, S. Starikov, V. Stegailov, V. Fortov, I. Skobelev, T. Pikuz, A. Faenov, S. Tamotsu, Y. Kato, M. Ishino, M. Tanaka, N. Hasegawa, M. Nishikino, T. Ohba, T. Kaihori, Y. Ochi, T. Imazono, Y. Fukuda, M. Kando, and T. Kawachi, "Nanomodification of gold surface by picosecond soft x-ray laser pulse," J. Appl. Phys. 112, 013104 (2012).

23. N. Medvedev, V. Tkachenko, V. Lipp, Z. Li, and B. Ziaja, "Various damage mechanisms in carbon and silicon materials under femtosecond x-ray irradiation," 4open 1, 3 (2018).

24. N. Medvedev, "Femtosecond $x$-ray induced electron kinetics in dielectrics: application for FEL-pulse-duration monitor," Appl. Phys. B 118, 417-429 (2015).
25. V. Lipp, N. Medvedev, and B. Ziaja, "Classical Monte Carlo simulations of x-ray induced electron cascades in various materials," Proc. SPIE 10236, 102360H (2017).

26. M. Kaganov, I. Lifshitz, and L. Tanatarov, "Relaxation between electrons and crystalline lattice," Sov. Phys. JETP 4, 173-178 (1957).

27. S. Anisimov, B. Kapeliovich, and T. Perelman, "Electron emission from metal surfaces exposed to ultrashort laser pulses," J. Exp. Theor. Phys. 66, 776-781 (1974).

28. D. E. Cullen, J. H. Hubbell, and L. Kissel, "EPDL97: the evaluated photon data library, '97 version," Technical Report UCRL-50400, Vol. 6 (Lawrence Livermore National Laboratory, 1997), Rev. 5.

29. B. Henke, E. Gullikson, and J. Davis, "X-ray interactions: photoabsorption, scattering, transmission, and reflection at $E=50-30,000 \mathrm{eV}$, Z = 1-92," At. Data Nucl. Data Tables 54, 181-342 (1993).

30. E. D. Palik, Handbook of Optical Constants of Solids, Academic Press Handbook Series (Academic, 1985), Vol. 1.

31. N. Medvedev, A. Volkov, and B. Ziaja, "Electronic and atomic kinetics in solids irradiated with free-electron lasers or swift-heavy ions," Nucl. Instrum. Methods Phys. Res. Sect. B 365, 437-446 (2015).

32. D. A. Chapman and D. O. Gericke, "Analysis of Thomson scattering from nonequilibrium plasmas," Phys. Rev. Lett. 107, 165004 (2011).

33. C. Jacoboni and L. Reggiani, "The Monte Carlo method for the solution of charge transport in semiconductors with applications to covalent materials," Rev. Mod. Phys. 55, 645-705 (1983).

34. T. M. Jenkins, W. R. Nelson, and A. Rindi, eds., Monte Carlo Transport of Electrons and Photons (Springer U.S., 1988).

35. Y.-K. Kim and M. E. Rudd, "Binary-encounter-dipole model for electron-impact ionization," Phys. Rev. A 50, 3954-3967 (1994).

36. N. Medvedev, "X-ray-induced electron cascades in dielectrics modeled with XCASCADE code: effect of impact ionization cross sections," Proc. SPIE 9511, 95110M (2015).

37. B. Rethfeld, A. Kaiser, M. Vicanek, and G. Simon, "Ultrafast dynamics of nonequilibrium electrons in metals under femtosecond laser irradiation," Phys. Rev. B 65, 214303 (2002).

38. R. R. Fäustlin, T. Bornath, T. Döppner, S. Düsterer, E. Förster, C. Fortmann, S. H. Glenzer, S. Göde, G. Gregori, R. Irsig, T. Laarmann, H. J. Lee, B. Li, K.-H. Meiwes-Broer, J. Mithen, B. Nagler, A. Przystawik, H. Redlin, R. Redmer, H. Reinholz, G. Röpke, F. Tavella, R. Thiele, J. Tiggesbäumker, S. Toleikis, I. Uschmann, S. M. Vinko, T. Whitcher, U. Zastrau, B. Ziaja, and T. Tschentscher, "Observation of ultrafast nonequilibrium collective dynamics in warm dense hydrogen," Phys. Rev. Lett. 104, 125002 (2010).

39. G. Chen, "Ballistic-diffusive heat-conduction equations," Phys. Rev. Lett. 86, 2297-2300 (2001).

40. N. Medvedev, Z. Li, V. Tkachenko, and B. Ziaja, "Electron-ion coupling in semiconductors beyond fermi's golden rule," Phys. Rev. B 95, 014309 (2017).

41. C. Lian, S. Zhang, and S. Meng, "Ab initio evidence for nonthermal characteristics in ultrafast laser melting," Phys. Rev. B 94, 184310 (2016).

42. H. Hu and S. A. Argyropoulos, "Mathematical modelling of solidification and melting: a review," Modell. Simul. Mater. Sci. Eng. 4, 371-396 (1996).

43. A. E. Morris, H. A. Fine, and G. Geiger, Handbook on Material and Energy Balance Calculations in Material Processing (Wiley, 2011).

44. S. Perkins, D. Cullen, M. Chen, J. Rathkopf, J. Scofield, and J. Hubbell, "Tables and graphs of atomic subshell and relaxation data derived from the LLNL evaluated atomic data library (EADL), $z=$ 1-100," Technical Report UCRL-50400-V-30 (Lawrence Livermore National Laboratory, 1991).

45. S. Gorbunov, N. Medvedev, P. Terekhin, and A. Volkov, "Electronlattice coupling after high-energy deposition in aluminum," Nucl. Instrum. Methods Phys. Res. Sect. B 354, 220-225 (2015).

46. D. A. Papaconstantopoulos, Handbook of the Band Structure of Elemental Solids: From $Z=1$ To $Z=112$ (Springer, 2016).

47. M. Bonn, D. N. Denzler, S. Funk, M. Wolf, S.-S. Wellershoff, and J. Hohlfeld, "Ultrafast electron dynamics at metal surfaces: competition between electron-phonon coupling and hot-electron transport," Phys. Rev. B 61, 1101-1105 (2000). 
48. B. Mueller and B. Rethfeld, "Relaxation dynamics in laser-excited metals under nonequilibrium conditions," Phys. Rev. B 87, 035139 (2013).

49. B. Rethfeld, D. S. Ivanov, M. E. Garcia, and S. I. Anisimov, "Modelling ultrafast laser ablation,” J. Phys. D 50, 193001 (2017).

50. S. I. Anisimov and B. Rethfeld, "Theory of ultrashort laser pulse interaction with a metal," Proc. SPIE 3093, 192-203 (1997).
51. C. Y. Ho, R. W. Powell, and P. E. Liley, "Thermal conductivity of the elements," J. Phys. Chem. Ref. Data 1, 279-421 (1972).

52. C. Kittel and P. McEuen, Introduction to Solid State Physics (Wiley, 1996), Vol. 8.

53. R. Ramji Rao and A. Ramanand, "Lattice dynamics, thermal expansion, and bulk modulus of ruthenium," J. Low Temp. Phys. 27, 837850 (1977). 